\title{
Cnidários do Mato Grosso do Sul, Brasil
}

\author{
William Marcos da Silva
}

Universidade Federal de Mato Grosso do Sul, Campus Pantanal, Av. Rio Branco, 1270, Vila Mamona, 79304-902, Corumbá, MS. (wmsilvax@ig.com.br)

Recebido 22 novembro 2016

Aceito 6 fevereiro 2017

DOI: $10.1590 / 1678-4766 e 2017103$

ABSTRACT. Cnidarians of Mato Grosso do Sul, Brazil. In this work it was a survey about the knowledge of the cnidarians in the state of Mato Grosso do Sul, and its studies possibilities for this group.

KEYWORDS. Hydrozoa, Craspedacusta sowerbii=sowerbyi, Brazilian Center-West, Biota-MS Program.

RESUMO. Neste trabalho foi levantado o conhecimento atual sobre os cnidários do Estado do Mato Grosso do Sul, e as possibilidades de estudos para este grupo.

PALAVRAS-CHAVE. Hydrozoa, Craspedacusta sowerbii=sowerbyi, centro-oeste brasileiro, Programa Biota-MS.

Filo representado por anêmonas, corais, pólipos e águas-vivas; é um grupo majoritariamente marinho, contendo aproximadamente $99,5 \%$ das espécies registradas e com poucos representantes em água doce - aproximadamente 23 espécies, distribuídas em 11 gêneros, entretanto com muitas incertezas taxonômicas (JANKOWSKI et al., 2008). Os cnidários dulceaquícolas estão todos incluídos em uma única classe, Hydrozoa, e distribuídos em duas subclasses: Hydoidolina contendo as formas de hidras solitárias sem metagênese e Trachylina que são representados por formas metagênicas (possuem alternância entre formas de pólipo e medusa) incluindo as formas parasitas (DALY et al. 2007; JANKOWSKI et al., 2008).

Os organismos deste filo possuem em comum as células denominadas cnidócitos, que secretam os nematocistos que contém substâncias complexas que auxiliam na alimentação e na defesa contra predadores. Estes organismos são essencialmente carnívoros, com algumas formas parasitas; em água doce se alimentam de pequenos invertebrados tais como microcrustáceos e rotíferos, eventualmente de ictioplâncton e são predados por turbelários, insetos aquáticos e crustáceos (Rocha, 1999). Algumas espécies de hidras podem ser pragas em pisciculturas (JANKOWSKI et al., 2008) e outras são simbiontes com algas Chlorella (SLOBODKIN \& BOSSERT, 2001).

Registros de espécies no Brasil e no Mato Grosso do Sul. Para o continente americano tem sido registrado aproximadamente 15 espécies de cnidários de água doce (JANKOWSKI et al., 2008) e sete no território brasileiro, distribuídos em todas as cinco regiões do país: Cordylophora caspia (Pallas, 1771), forma hidróide colonial comum em estuários; Hydra viridissima Pallas, 1766, Hydra iheringi Cordero, 1939, Hydra intermedia Wolle, 1978, Hydra salmacidis Silveira et al., 1997, formas hidroides solitárias; Calpasoma dactyloptera Furhman, 1939, Microhydra ryderi Potts, 1906, Craspedacusta sowerbii=sowebyi Lankester, 1880, formas metagênicas (Silveira \& SCHLEnZ, 1999).

No estado de Mato Grosso do Sul, e na região CentroOeste, apenas Craspedacusta sowerbii $=$ sowebyi Lankester, 1880 na forma de medusa foi registrada na bacia do Rio Miranda em uma dolina (lagoa Misteriosa) no município de Jardim (SiLVA \& Roche, 2007) (Fig. 1). Apesar do único registro, há relatos de blooms de águas-vivas em outros corpos de águas desta região; evidentemente a forma pólipo

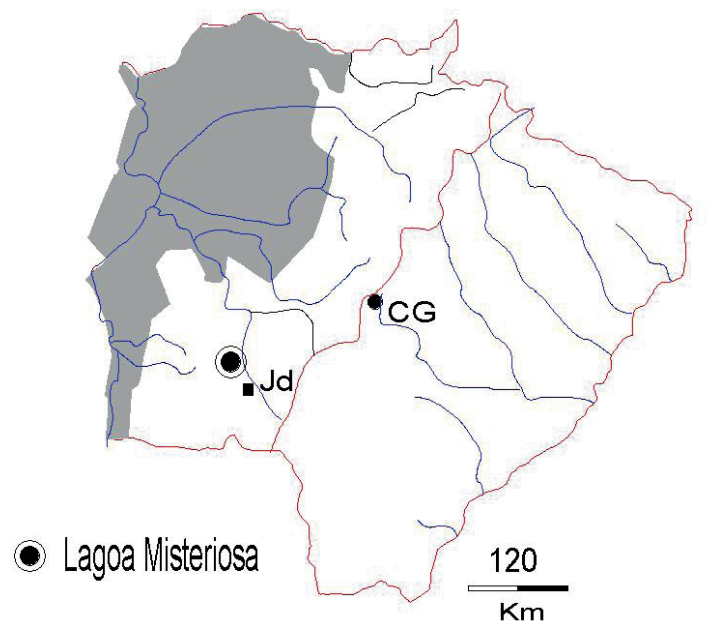

Fig. 1. Localização da Lagoa Misteriosa no estado do Mato Grosso do Sul, Brasil (Jd, município de Jardim; CG, município de Campo Grande). 
desta espécie está presente nos corpos de água, entretanto o tamanho diminuto do pólipo com cerca de $1 \mathrm{~mm}$ dificulta a visualização e até mesmo o reconhecimento.

Principais grupos de pesquisa no Estado. $\mathrm{O}$ estudo de Cnidaria no Mato Grosso do Sul é ainda insipiente, tanto em sua taxonomia, como na biologia e ecologia e não conta com grupos de pesquisa nem pesquisadores especialistas e não há coleções científicas, apenas organismos em coleções didáticas nos laboratórios de microbiologia ambiental na UFMS, campus Campo Grande e no Laboratório de Ecologia na UFMS, Campus Pantanal, Corumbá. Apesar de não haver pesquisadores especialistas no Filo Cnidaria, existem pesquisadores em áreas com potencial para estudos de distribuição e de ecotoxicologia: Prof. Dr. William Marcos da Silva, UFMS Campus Pantanal, Corumbá e Prof. Dr. Kennedy Francis Roche, UFMS Campus Campo Grande.

Perspectivas de Pesquisas. Os cnidários de água doce têm sido utilizados como indicadores de eutrofização de sistemas aquáticos (RocHA, 1999) e de toxicidade ambiental e qualidade de água (MASSARo \& RoCHA, 2008; MASSARO 2011; QuinN et al., 2012). Estudos ecotoxicológicos estão sendo iniciados no curso de Pós-Graduação de Tecnologias Ambientais na UFMS, Campo Grande (NogueIRA, 2010), onde Hydra sp. é cultivada para a realização de bioensaios para fins ecotoxicológicos. O conhecimento da distribuição geográfica deste grupo no estado poderia ser ampliado através de treinamento de recursos humanos que estudam a fauna de macroinvertebrados bentônicos.

Agradecimentos. A Fundação de Apoio ao Desenvolvimento do Ensino, Ciências e Tecnologia do Estado de Mato Grosso do Sul (FUNDECT) e a Superintendência de Ciências e Tecnologia do Estado de Mato Grosso do Sul (SUCITEC/MS) pelo convite de participação neste volume especial da Iheringia, Série Zoologia e o suporte financeiro para sua publicação.

\section{REFERÊNCIAS BIBLIOGRÁFICAS}

Daly, M.; Brugler, M. R.; Cartwright, P.; Collins, A. G.; Dawson, N. M.; Fautin, D. G.; France, S. C.; McFadden, C. S.; Opresko, D. M.; Rodriguez, E.; Romano, S. L. \& Stake, J. L. 2007. The phylum Cnidaria: A review of phylogenetic patterns and diversity 300 years after Linnaeus. Zootaxa 1668:127-182.

Jankowski, T.; Collins, A. G. \& Campbell, R. 2008. Global diversity of inland water cnidarians. Hydrobiologia 595:35-40.

MASSARO, F. C. 2011. Estudos ecológicos e ecotoxicológicos de espécies nativas de Hydra (Cnidaria: Hydrozoa). Tese doutorado. São Carlos, Escola de Engenharia de São Carlos, Universidade de São Paulo. 414p.

Massaro, F. C. \& Rocha, O. 2008. Development and population growth of Hydra viridissima Pallas, 1766 (Cnidaria, Hydrozoa) in the laboratory. Brazilian Journal of Biology 68(2):379-383.

Nogueira, T. D. 2010. Efeitos Agudos de Efluentes Líquidos Industriais. Dissertação de Mestrado. Campo Grande, Pós-Graduação em Tecnologias Ambientais, Universidade Federal de Mato Grosso do Sul.

Quinn, B.; Gagné, F. \& Blaise, C. 2012. Hydra, a model system for environmental studies. The International Journal of Developmental Biology 56:613-625.

Rocha, O. 1999. Organismos de água doce. In: Lewinsohn, T. M. \& Prado, P. I. (Eds.). Síntese do conhecimento atual da biodiversidade brasileira. São Paulo, Contexto Acadêmica.172p.

SiLVA, W. M. \& Roche, K. F. 2007. Occurrence of the freshwater jellyfish Craspedacusta sowerbii (Lankester, 1880) (Hydrozoa, Limnomedusae) in a calcareous lake in Mato Grosso do Sul, Brazil. Biota Neotropica 7. Disponível em: <http://www.biotaneotropica.org.br/v7n1/pt/ abstract?short-communication+bn02107012007>.

Silveira, F. L. \& Schlenz, E. 1999. Cnidários. In: Ismael, D.; Valentini, W. C.; Matsumura-Tundisi, T. \& Rocha, O. eds. Biodiversidade do estado de São Paulo, Brasil: invertebrados de água doce. São Paulo, Fundação de Amparo à Pesquisa do Estado de São Paulo. p. 11-15.

Slobodkin, L. B. \& Bossert, P. E. 2001. Cnidaria. In: Thorp, J. H. \& Covith, A. P. eds. Ecology and Classification of North American Freshwater Invertebrates. 2ed. San Diego, Academy Press. 1056p. 